\title{
microRNA expression profile and differentially-expressed genes in prolactinomas following bromocriptine treatment
}

\author{
CHENGDE WANG $^{1 *}$, ZHIPENG SU $^{1 *}$, NADER SANAI $^{2}$, XIANGYANG XUE $^{3}$, LIJUN LU $^{4}$, \\ YUNXIANG CHEN $^{1}$, JINSEN WU ${ }^{1}$, WEIMING ZHENG ${ }^{1}$, QICHUAN ZHUGE $^{1}$ and ZHE BAO WU ${ }^{1}$ \\ ${ }^{1}$ Department of Neurosurgery, First Affiliated Hospital of Wenzhou Medical College, Wenzhou 325000; \\ ${ }^{2}$ Barrow Brain Tumor Research Center, Barrow Neurological Institute, Phoenix, AZ 85013, USA; ${ }^{3}$ Department \\ of Microbiology and Immunology, Wenzhou Medical College, Wenzhou 325000; ${ }^{4}$ Key Laboratory of \\ Surgery, First Affiliated Hospital of Wenzhou Medical College, Wenzhou 325000, P.R. China
}

Received November 18, 2011; Accepted January 2, 2012

DOI: 10.3892/or.2012.1690

\begin{abstract}
Little is known about the function of microRNAs in prolactinomas treated with bromocriptine. The aim of the study was to explore the microRNAs associated with bromocriptinetreated prolactinomas. Six prolactinoma samples were selected according to whether they received bromocriptine treatment or not before microsurgery, and microRNA expression profiles of bromocriptine-treated and untreated prolactinomas were screened by the miRCURY LNA Array. The differentially expressed microRNAs in microarrays were further validated by stem-loop real-time PCR and subjected to gene ontology analysis and KEGG pathway analysis. In addition, related genes of microRNAs were analyzed by qRT-PCR in 15 prolactinoma samples. The initial analysis by microarrays generated a list of 80 upregulated microRNAs and 71 downregulated microRNAs in treated prolactinomas compared to untreated prolactinomas. miR-206, miR-516b and miR-550 were confirmed to be significantly upregulated, while miR-671-5p was confirmed to be significantly downregulated in treated prolactinomas by qRT-PCR. microRNA-mRNA network analysis integrating GO and KEGG pathway annotation displayed some critical
\end{abstract}

Correspondence to: Dr Zhe Bao Wu, Department of Neurosurgery, First Affiliated Hospital of Wenzhou Medical College, Wenzhou 325000, P.R. China

E-mail: zhebaowu@yahoo.com.cn

${ }^{*}$ Contributed equally

Abbreviations: mRNA, messenger RNA; NFPA, non-functioning pituitary adenoma; PCR, polymerase chain reaction; qRT-PCR, semi-quantitative real-time-PCR; PRL, prolactin; BMP4, bone morphogenetic protein 4; PDGFRA, platelet-derived growth factor receptor $\alpha$ polypeptide; PDGFA, platelet-derived growth factor $\alpha$ polypeptide; BMPR2, bone morphogenetic protein receptor type II

Key words: microRNAs, prolactinoma, bromocriptine, PDGFA, microarray factors. Platelet-derived growth factor $\alpha$ polypeptide (PDGFA) and bone morphogenetic protein 4 (BMP4), were verified to be differentially expressed between the two groups. PDGFA was significantly upregulated in treated prolactinomas, while BMP4 was significantly downregulated in treated prolactinomas. Our study reveals differential expression of microRNAs in prolactinoma after pharmacotherapy. Specific microRNAs may be involved in the inhibition or promotion of prolactinoma tumor growth impacted by bromocriptine pharmacotherapy. PDGFA and BMP4 may be involved in the pharmacotherapy mechanism of prolactinoma.

\section{Introduction}

Dopamine agonist medication is a first-line therapy in the treatment of prolactinomas. Bromocriptine can normalize the serum prolactin (PRL) level in more than $90 \%$ of cases and reduce tumor volume in approximately $85 \%$ of cases (1). Its primary mechanism of action is through the dopamine 2 (D2) receptor on the cell membrane of prolactin cells, which is selectively activated. Thus, the transcription and expression of the PRL gene and the metabolism of prolactin cells are inhibited, leading to decreased synthesis and secretion of PRL. In addition, the involution of the endoplasmic reticulum and Golgi apparatus, and the suppression of cell proliferation lead to a reduction in tumor volume (2). The subsequent pharmacotherapeutic effect of prolactinoma acts through the TGF- $\beta$ and MAPK signaling pathway to inhibit lactotroph cell proliferation and induce apoptosis through D2 receptors (3-5).

microRNAs are approximately 22 nucleotide, non-coding RNA generated from endogenous hairpin-shaped transcripts which post-transcriptionally regulate the expression of protein-coding genes (6) and are thought to be involved in several cellular processes regulating differentiation and development $(7,8)$. microRNAs may regulate gene expression at the post-transcriptional level by direct cleavage of a target mRNA using interference machinery (mRNA cleavage) or by inhibition of protein synthesis, thereby affecting the expression of several hundred different target genes (9). microRNAs bind to the 3'-untranslated region of target mRNAs, interrupting translation or mRNA degradation (6). They are involved in 
Table I. Patient characteristics of treated and untreated prolactinomas with bromocriptine.

\begin{tabular}{|c|c|c|c|c|c|c|c|}
\hline Case no. & Group & Gender & Age & $\begin{array}{l}\text { Initial PRL } \\
\text { (ng/ml) }\end{array}$ & Tumor size & $\begin{array}{l}\text { BRC daily doses } \\
\text { (mg) }\end{array}$ & $\begin{array}{c}\text { Time with BRC } \\
\text { (months) }\end{array}$ \\
\hline $1^{\mathrm{a}}$ & A & $\mathrm{F}$ & 35 & 872.5 & Macro+KnospI+SSE & 5 & 2 \\
\hline $2^{\mathrm{a}}$ & $\mathrm{A}$ & M & 43 & $>400$ & Macro+KnospII+SSE & 5.0 & 15 \\
\hline $3^{\mathrm{a}}$ & A & $\mathrm{F}$ & 39 & $>200$ & Micro & 5 & 18 \\
\hline 4 & A & $\mathrm{F}$ & 27 & $>200$ & Macro+KnospIV+SSE & 7.5 & 7 \\
\hline 5 & A & $\mathrm{F}$ & 35 & $>200$ & Macro+KnospII+SSE & 7.5 & 20 \\
\hline $6^{\mathrm{a}}$ & $\mathrm{B}$ & M & 45 & 849 & Macro+KnospII+SSE & & \\
\hline $7^{\mathrm{a}}$ & B & M & 51 & $>200$ & Macro+KnospI & & \\
\hline $8^{\mathrm{a}}$ & $\mathrm{B}$ & $\mathrm{M}$ & 43 & $>200$ & Macro+KnospII+SSE & & \\
\hline 9 & $\mathrm{~B}$ & $\mathrm{~F}$ & 47 & 287.5 & Micro & & \\
\hline 10 & B & M & 42 & 1733.6 & Macro+KnospIV+SSE & & \\
\hline 11 & $\mathrm{~B}$ & M & 47 & $>4000$ & Macro+KnospII+SSE & & \\
\hline 12 & $\mathrm{~B}$ & M & 23 & 3814 & Macro+KnospIV+SSE & & \\
\hline 13 & $\mathrm{~B}$ & $\mathrm{~F}$ & 52 & $>600$ & Macro+KnospIV+SSE & & \\
\hline 14 & $\mathrm{~B}$ & M & 38 & $>200$ & Macro+KnospII+SSE & & \\
\hline 15 & $\mathrm{~B}$ & M & 29 & $>800$ & Macro+KnospIV & & \\
\hline
\end{tabular}

PRL, prolactin; F, female; M, male; BRC, bromocriptine; Macro, macroadenoma; Micro, microadenoma; SSE, supraseller extension. ${ }^{\text {aSix }}$ patient samples were screened using the miRCURY ${ }^{\mathrm{TM}}$ LNA Array. Patient cases 1,2 and 3 were treated with bromocriptine and cases 6,7 and 8 were not treated with bromocriptine before microsurgery.

several other cellular processes, including differentiation, proliferation and death (8). Specific microRNA enrichment has been described in the adult mouse pituitary, suggesting that microRNAs may regulate pituitary functions (10-12) and development (13). Recently, a number of studies have analyzed the microRNA expression in pituitary adenomas (14-18). Bottoni et al (14) found that miR-15a and miR-16-1 dysregulation may affect the expression of genes involved in the development of pituitary adenomas. Furthermore, Bottoni et al (15) analyzed the entire microRNAome in pituitary adenomas and normal pituitary and found that specific microRNAs were identified as a predictive signature of pituitary adenoma and its histotype. In addition, miR-135a, miR-140-5p, miR-582-3p, miR-582-5p and miR-938, which target Smad3 (19), and miR-128a, miR-155 and miR-516a-3p, which target Weel (20), were overexpressed in non-functioning pituitary adenomas. Furthermore a group set of microRNAs differentially expressed among normal pituitaries adenomas and carcinomas has been described (21). However, in the current literature, no investigation on the alteration of microRNA expression in prolactinomas undergoing pharmacological treatment by bromocriptine has been performed despite the fact that microRNA expression could differentiate treated from non-treated NFPA samples in the Bottoni's report (16). In this study, we screen and identified microRNAs associated with prolactinomas treated with bromocriptine, and further analyzed the related genes that are also involved in the pharmacotherapeutic mechanism of prolactinoma. The present findings may provide a basis to comprehend their potential role in the pharmacotherapeutic mechanism of prolactinoma.

\section{Patients and methods}

Patients. This study was approved by the Ethics Committee of Wenzhou Medical College, and written informed consent was obtained from all patients. Fifteen postsurgical pituitary adenoma tissue samples were obtained from the First Affiliated Hospital of Wenzhou Medical College; data from 6 of those patients have been previously reported (22) (Table I). Tissue samples were collected in accordance with the guidelines of the local committee on human research. Patients were classified into 2 groups according to whether they received bromocriptine treatment or not before microsurgery. Five patients of Group A received bromocriptine treatment before microsurgery: one patient was not able to tolerate the side effects, 3 patients were reluctant to take bromocriptine for life, and one patient underwent tumor apoplexy during the bromocriptine treatment. In order to eliminate the adverse effects of different doses and durations of bromocriptine treatment, bromocriptine, no less than $5 \mathrm{mg}$ per day, was taken for at least 8-weeks in this study (Table I). Ten patients (Group B) primarily received microsurgery (5 patients with visual impairment, 1 with tumor apoplexy, and 4 with an individual decision for a primarily surgical treatment). In each patient, the diagnosis of a prolactinoma was confirmed by tumor histopathological, immunohistochemical and electron microscopy evaluation.

RNA isolation and evaluation. Total RNA was extracted from pituitary adenoma tissues using TRIzol reagent (Invitrogen Life Technologies, Carlsbad, CA, USA) according to the 
manufacturer's instructions with some modifications as previously described (23). RNAs were incubated at $-20^{\circ} \mathrm{C}$ for at least $2 \mathrm{~h}$ to enhance precipitation efficiency of low-molecular-weight (LMW) RNAs. Following a wash with $80 \%$ ethanol, RNA was resuspended in DEPC-treated water and stored at $-80^{\circ} \mathrm{C}$. The concentration and purity of total RNA were qualified by an ultraviolet spectrophotometer at 260 and $280 \mathrm{~nm}$.

microRNA microarray. Six high-density expression arrays were used (Group A patient cases 1, 2 and 3; Group B patient cases 6, 7 and 8) (Table I) to analyze the microRNA expression. The samples were labeled using the miRCURY ${ }^{\mathrm{TM}} \mathrm{Hy} 3^{\mathrm{TM}} /$ $\mathrm{Hy}^{\mathrm{TM}}$ Power labeling kit and hybridized on miRCURY LNA Array (v.10.0) containing capture probes for 856 microRNAs, listed in miRBase (Sanger miRBase Release 10.0) http:// microrna.sanger.ac.uk). A common reference consisting of a mix of the products was spotted on the array. Accordingly, a ratio can be calculated for every spot, and expression levels across different hybridizations can be compared. This approach provides a reference point that is durable for many hybridizations (24). Scanning is performed with the Axon GenePix 4000B microarray scanner. The ratio of green signal to red signal is calculated after background subtraction and normalization using the global Lowess (Locally Weighted Scatter plot Smoothing) regression algorithm (MIDAS, TIGR Microarray Data Analysis System), which was found to produce the best within-slide normalization to minimize the intensity-dependent differences between the dyes (http:// www.exiqon.com). The positive effect of this normalization is illustrated in 2 different plots for each slide. The Cy3/Cy5 ratios were calculated as the treated group/untreated group signals $\mathrm{x} \log 2$. The statistical significance of differentially expressed microRNA was analyzed by t-test.

Microarray data accession number. The microarray data discussed in this study have been deposited in the NCBI's Gene Expression Omnibus (GEO; http://www.ncbi.nlm.nih. gov/geo/) and are accessible through GEO series accession number GSE23927 (http://www.ncbi.nlm.nih.gov/geo/query/ acc.cgi?acc=GSE23927). All data in this study are MIAME compliant.

Quantification of the microRNA expression in prolactinoma specimens. Stem-loop real-time RT-PCR (qRT-PCR) was used in the analysis of microRNA expression. The primers for the analysis of microRNAs expression were designed according to Chen et al (25). Mixtures of $1 \mu \mathrm{g}$ of total RNAs together with $50 \mathrm{nM}$ reverse primer, 2 units of the RNAase inhibitor (Toyobo, Osaka, Japan), 5 units of M-MLV reverse transcriptase (Toyobo) and $0.5 \mu \mathrm{M}$ dNTP were used for each $\mathrm{RT}$ reaction. The internal control U6 was reverse transcribed in parallel. The reactions were incubated for $30 \mathrm{~min}$ at $16^{\circ} \mathrm{C}$, $30 \mathrm{~min}$ at $42^{\circ} \mathrm{C}, 15 \mathrm{~min}$ at $70^{\circ} \mathrm{C}$ and then held at $4^{\circ} \mathrm{C}$. The qRT-PCR reaction was performed on the Applied Biosystems 7500 detection system by a $20 \mu 1$ reaction system (Toyobo) including $10 \mu \mathrm{l}$ SYBR-Green Real-time PCR Master Mix-plus, $2 \mu$ l Plus solution, $2 \mu \mathrm{l}$ each specific forward and reverse primers, $1 \mu \mathrm{l}$ RT product of total RNA and $3 \mu \mathrm{l}$ DEPC water. The reaction was performed at $95^{\circ} \mathrm{C}$ for $2 \mathrm{~min}$, followed by 40 amplification cycles at $95^{\circ} \mathrm{C}$ for $15 \mathrm{sec}$ and $60^{\circ} \mathrm{C}$ for $1 \mathrm{~min}$.
Melting curves were generated for each real-time RT-PCR to verify the specificity of each PCR reaction. The Ct (threshold cycle) value is defined as the fractional cycle number at which the fluorescence passed the fixed threshold. $\Delta \mathrm{Ct}$ represents the expression difference between the target microRNA and the normalizer: $\Delta \mathrm{Ct}=\mathrm{Ct}$ (mir)- $\mathrm{Ct}$ (normalizer). The $\Delta \Delta \mathrm{Ct}$ method recommended by the manufacturer was used to compare relative expression levels between the groups.

Quantification of gene expression in prolactinoma specimens. cDNA synthesis was carried out via ReverTra Ace reverse transcriptase (Toyobo) with oligo (dT)18 primers. Semiquantitative PCR was performed with various forward and reverse primers. qRT-PCR was carried out using SYBR-Green RT-PCR kit (Toyobo) and was performed on the Applied Biosystems 7500 detection system. Each PCR cycle included $94^{\circ} \mathrm{C}$ for $30 \mathrm{sec}, 60^{\circ} \mathrm{C}$ for $30 \mathrm{sec}$ and $72^{\circ} \mathrm{C}$ for $1 \mathrm{~min}$. GAPDH was used as the internal control. Similarly, $\Delta \mathrm{Ct}$ represents the expression difference between the target gene and the normalizer: $\Delta \mathrm{Ct}=\mathrm{Ct}($ gene $)-\mathrm{Ct}($ normalizer). The normalized gene expression in a sample was expressed with $2^{-\Delta \Delta C t}$.

Identification of predicted miRNA targets, gene ontology category and pathway analysis. The predicted target mRNAs of 4 miRNAs (miR-206, miR-516b, miR-550 and miR-671-5p) were performed by search algorithms, TargetScan (http://www. targetscan.org/). These putative target genes were loaded to DAVID Bioinformatics Resources 6.7 (26) where GO analysis and KEGG pathway analysis were applied in order to uncover the miR-Gene Regulatory Network. In detail, two-side Fisher's exact tests were used to classify the GO category and KEGG pathway, and the false discovery rate (FDR) was calculated to correct the P-value. P-value $<0.05$ and FDR $<0.05$ were used as a threshold to select significant GO categories and KEGG pathways.

Statistical analysis. As the data for miRNA and gene relative expression levels did not fit a Gaussian distribution, the expression levels were characterized by their median and ranges from the 25th to the 75th percentile, rather than their mean and coefficient of variation. A non-parametric test (Mann-Whitney $\mathrm{U}$ test) was used to evaluate the difference of microRNA and relative gene expression between treated and untreated prolactinomas with bromocriptine. $\mathrm{P}<0.05$ was considered to indicate a significant difference. Correlation between the expression of microRNAs and target genes in human pituitary adenomas was confirmed using the Spearman's correlation coefficient analyses. All calculations were performed with the software SPSS 13.0.

\section{Results}

microRNAs differentially expressed in treated and untreated prolactinomas with bromocriptine by microarray. In order to reduce the false negative rate, we considered a 1.2-fold-change and P-value of 0.1 as the necessary indicators according to this microarray data. The initial analysis of microRNA expression data generated a list of 71 microRNAs with a 1.2-fold lower expression in treated prolactinomas compared with untreated tumors, of which 6 microRNAs were downregulated in treated 
Table II. microRNAs differentially expressed in treated and untreated prolactinomas with bromocriptine by microarray.

\begin{tabular}{lcccr}
\hline & \multicolumn{3}{c}{ Mean \pm SD } & \\
miRNA & Group A (treated) & Group B (untreated) & Group A/Group B & P-value \\
\hline miR-206 & $1.192 \pm 0.058$ & $0.938 \pm 0.046$ & 1.26 & 0.014 \\
miR-516b & $1.191 \pm 0.066$ & $0.923 \pm 0.137$ & 1.28 & 0.080 \\
miR-630 & $1.186 \pm 0.049$ & $0.966 \pm 0.104$ & 1.22 & 0.076 \\
miR-617 & $1.184 \pm 0.081$ & $0.979 \pm 0.078$ & 1.20 & 0.096 \\
miR-576-3p & $1.263 \pm 0.164$ & $0.833 \pm 0.154$ & 1.51 & 0.075 \\
miR-374b & $1.136 \pm 0.047$ & $0.913 \pm 0.083$ & 1.24 & 0.053 \\
miR-516a-5p & $1.200 \pm 0.066$ & $0.955 \pm 0.110$ & 1.25 & 0.075 \\
miR-488 & $1.216 \pm 0.168$ & $0.867 \pm 0.096$ & 1.40 & 0.092 \\
miR-550 & $1.212 \pm 0.060$ & $0.905 \pm 0.169$ & 1.34 & 0.096 \\
miR-491-5p & $0.833 \pm 0.096$ & $1.342 \pm 0.096$ & 0.62 & 0.064 \\
miR-487b & $0.697 \pm 0.131$ & $1.389 \pm 0.055$ & 0.50 & 0.040 \\
miR-S1-5p & $0.855 \pm 0.107$ & $1.472 \pm 0.336$ & 0.58 & 0.082 \\
miR-585 & $0.797 \pm 0.160$ & $1.227 \pm 0.134$ & 0.64 & 0.064 \\
miR-671-5p & $0.608 \pm 0.153$ & $1.081 \pm 0.112$ & 0.56 & 0.031 \\
miR-377 & $0.866 \pm 0.048$ & $1.100 \pm 0.032$ & 0.78 & 0.006 \\
\hline
\end{tabular}

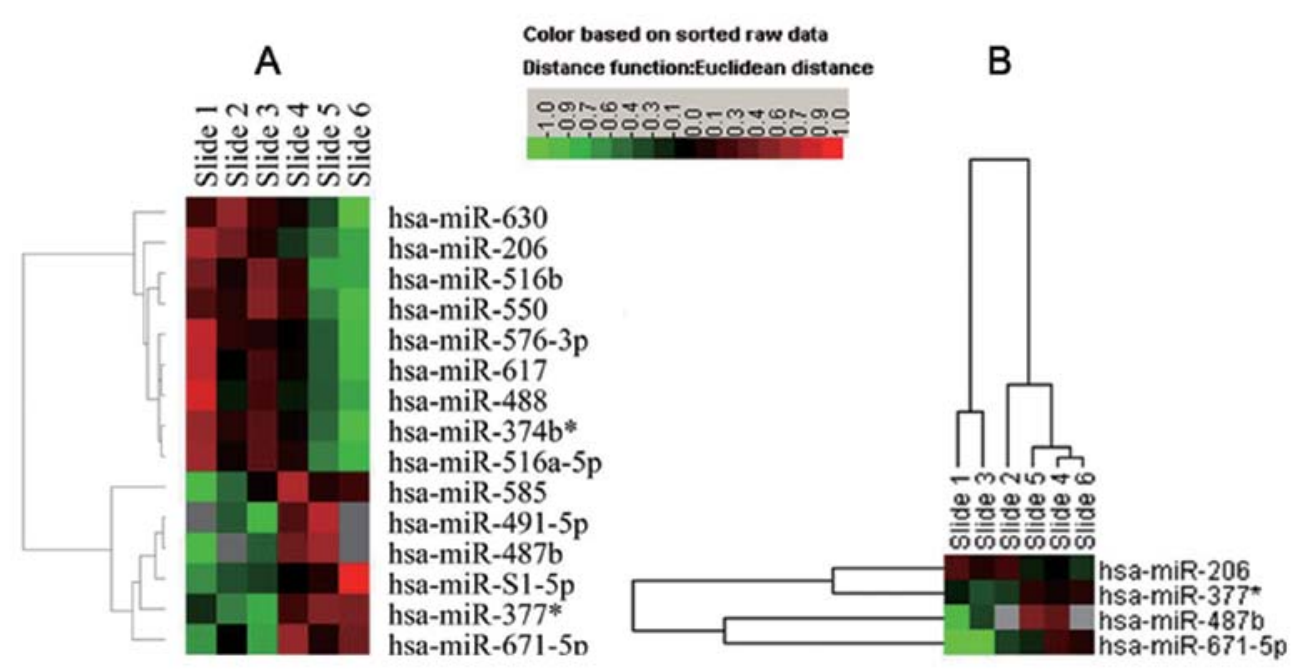

Figure 1. Heat Map shows an unsupervised hierarchical clustering result of microRNAs. In the Cy3/Cy5 ratio image, red denotes a higher expression level than in the common reference; green, lower expression level than the common reference. In the lower table, each line represents a miRNA, and each row represents a sample (slide). (A) Fifteen microRNAs differentially expressed between the treated and untreated prolactinomas with a P-value $<0.1$. (B) Nine microRNAs differently expressed between the treated and untreated prolactinomas with a P-value $<0.05$.

prolactinomas with a P-value $<0.1$. Inversely, the data also generated a list of 80 microRNAs, with 1.2-fold or higher expression in treated prolactinomas compared with untreated tumors, of which 9 microRNAs were upregulated in treated prolactinomas with a P-value $<0.1$ (Table II; Fig. 1A). Among these 15 microRNAs, 4 microRNAs were found to be significantly differentially expressed with a P-value $<0.05$ between the 2 groups by microarray; 3 of these were upregulated and the other was downregulated (Table II; Fig. 1B).

Validation of differentially expressed microRNAs by semiquantitative RT-PCR. In order to further validate microarray results, we performed qRT-PCR expression analysis for the
15 differentially expressed microRNAs whose P-value was $<0.1$ by microarray (Table III). The expression analysis of these selected microRNAs was carried out on specimens from the 15 patients (Table I). Melting curves were generated for each real-time RT-PCR to verify the specificity of each PCR reaction. All the 15 mature microRNAs were expressed in 15 prolactinoma samples. Expression of microRNAs was normalized with respect to U6 gene expression and by using the relative quantification approach previously described (27). Relative expression of 3 specific microRNAs, miR-206, miR-516b and miR-550 were significantly higher in the treated prolactinomas compared to untreated prolactinomas ( $\mathrm{P}=0.029, \mathrm{P}=0.006$ and $\mathrm{P}=0.024$ respectively) (Table III and 
Table III. microRNAs differentially expressed in treated and untreated prolactinomas with bromocriptine by RT-PCR.

\begin{tabular}{lccr}
\hline miRNAs & Group A (treated) median $(\mathrm{p} 25, \mathrm{p} 75)$ & Group B (untreated) median $(\mathrm{p} 25, \mathrm{p} 75)$ & P-value \\
\hline miR-206 & $0.75(0.70,0.90)$ & $0.55(0.48,0.69)$ & 0.029 \\
miR-516b & $1.61(1.10,1.82)$ & $0.30(0.94,0.61)$ & 0.006 \\
miR-630 & $1.00(0.43,369.09)$ & $0.28(0.75,1.18)$ & 0.165 \\
miR-617 & $1.44(0.91,26.05)$ & $0.77(0.24,1.41)$ & 0.171 \\
miR-576-3p & $0.51(0.24,12.61)$ & $0.25(0.23,0.42)$ & 0.165 \\
miR-374b & $0.75(0.16,0.93)$ & $0.59(0.39,0.87)$ & 0.953 \\
miR-516a-5p & $4.93(0.67,8.95)$ & $0.94(0.50,3.37)$ & 0.310 \\
miR-488 & $0.19(0.05,0.74)$ & $0.25(0.06,1.02)$ & 0.859 \\
miR-550 & $0.75(0.30,0.34)$ & $0.45(0.14,0.52)$ & 0.024 \\
miR-491-5p & $0.05(0.01,0.77)$ & $0.46(0.21,1.60)$ & 0.635 \\
miR-487b & $0.62(0.33,1.20)$ & $0.47(0.32,1.62)$ & 0.833 \\
miR-s1-5p & $0.95(0.31,7.12)$ & $0.45(0.21,1.60)$ & 0.635 \\
miR-585 & $2.65(0.69,5.42)$ & $0.98(0.23,3.65)$ & 0.374 \\
miR-671-5p & $1.24(0.87,1.85)$ & $2.66(1.62,2.92)$ & 0.042 \\
miR-377 & $0.79(0.62,1.35)$ & $0.65(0.40,1.09)$ & 0.310 \\
\hline
\end{tabular}
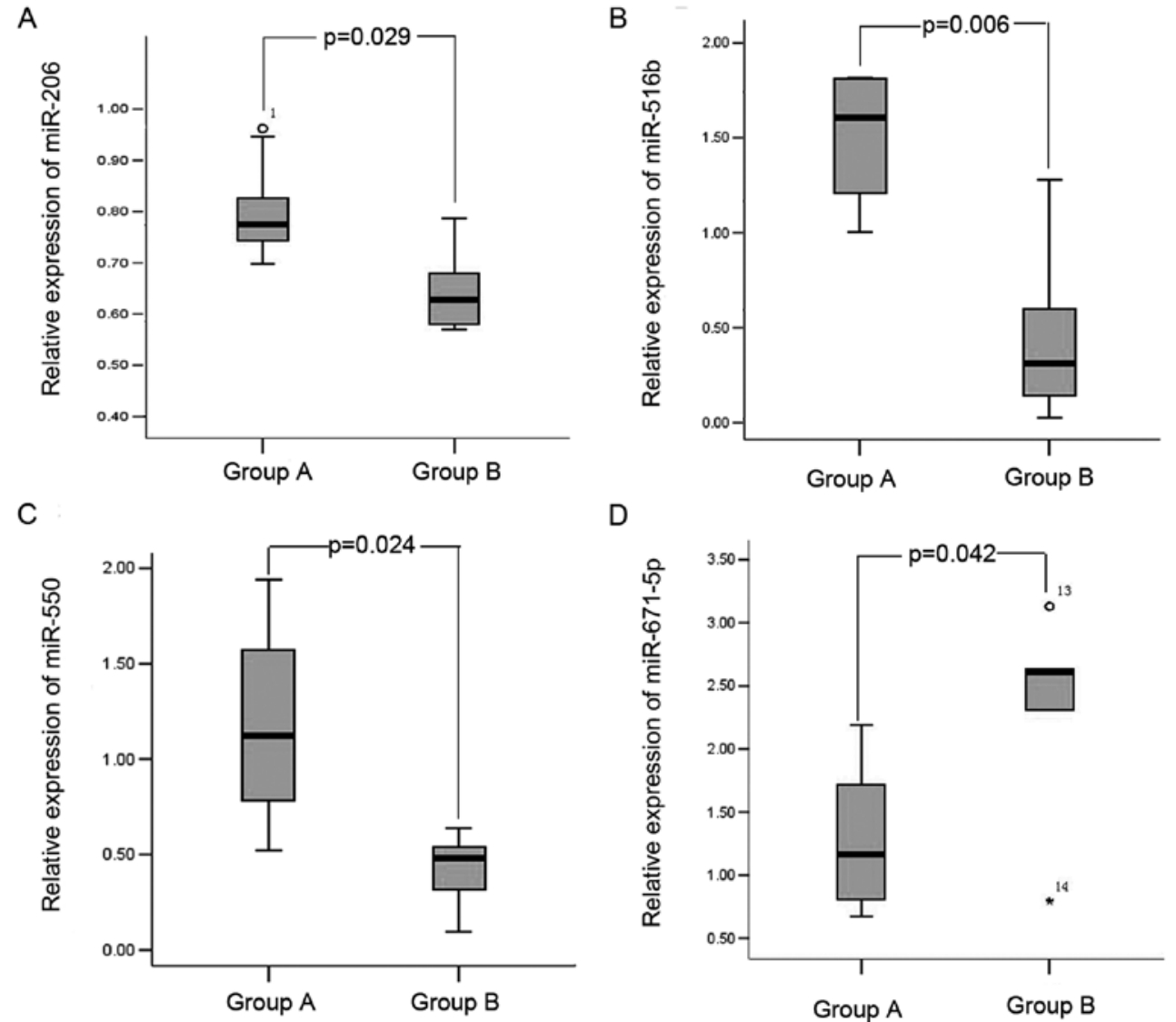

Figure 2. Four microRNAs differentially expressed between treated and untreated prolactinomas by RT-PCR. (A) Boxplots showing significantly higher expression of (A) miR-206 ( $\mathrm{P}=0.029)$; (B) miR-516b ( $\mathrm{P}=0.006)$ and (C) miR-550 ( $\mathrm{P}=0.024)$; boxplot showing significantly lower expression of (D) miR-671-5p $(\mathrm{P}=0.042)$.

Fig. 2A-C). For another microRNA, miR-671-5p, the relative expression was significantly lower in treated prolactinomas ( $\mathrm{P}=0.042)$ (Fig. 2D).
Gene ontology $(G O)$ category and pathway analysis. Total putative target genes (1096) for miR-206, miR-516b, miR-550 and miR-671-5p were found by TargetScan (data not shown). 
A

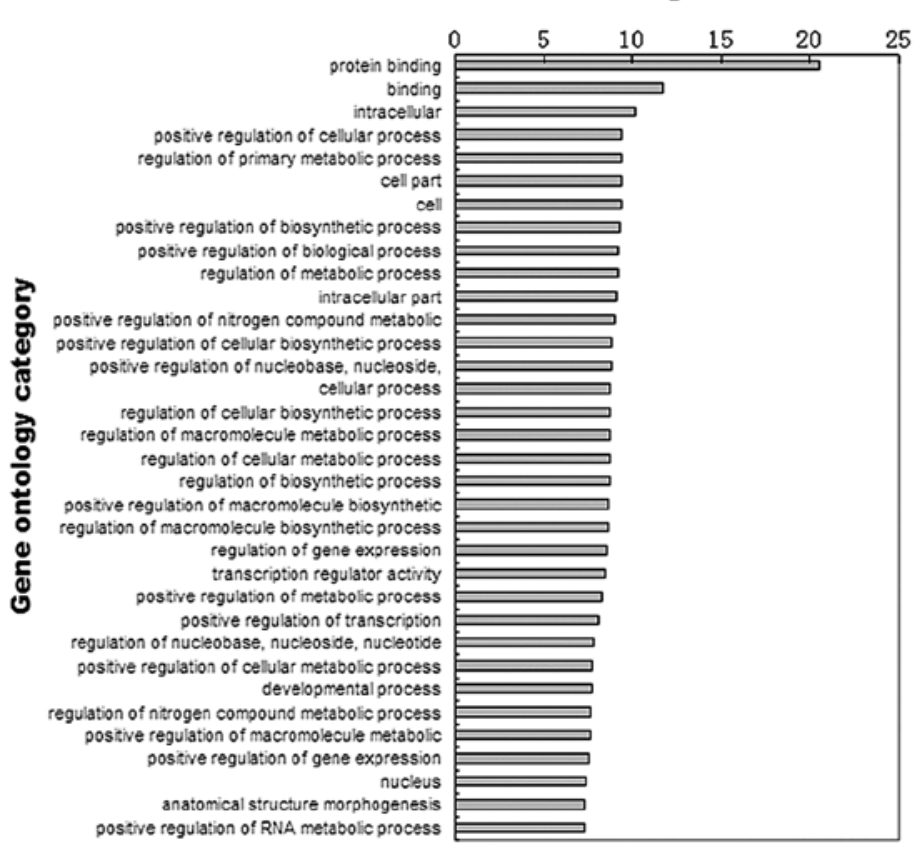

B

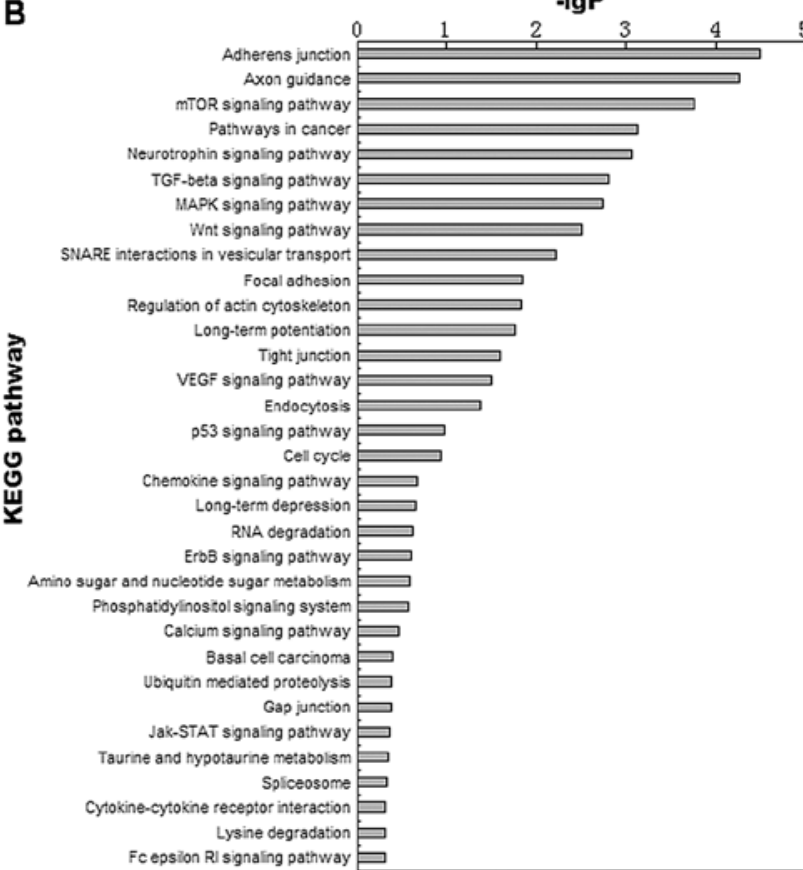

Figure 3. (A) GO category for putative target genes. P-value $<0.05$ and FDR $<0.05$ were used as a threshold to select significant GO categories. (B) KEGG pathway analysis for putative target genes. P-value $<0.05$ and FDR $<0.05$ were used as a threshold to select significant KEGG pathways; lgP is the negative logarithm of the P-value.

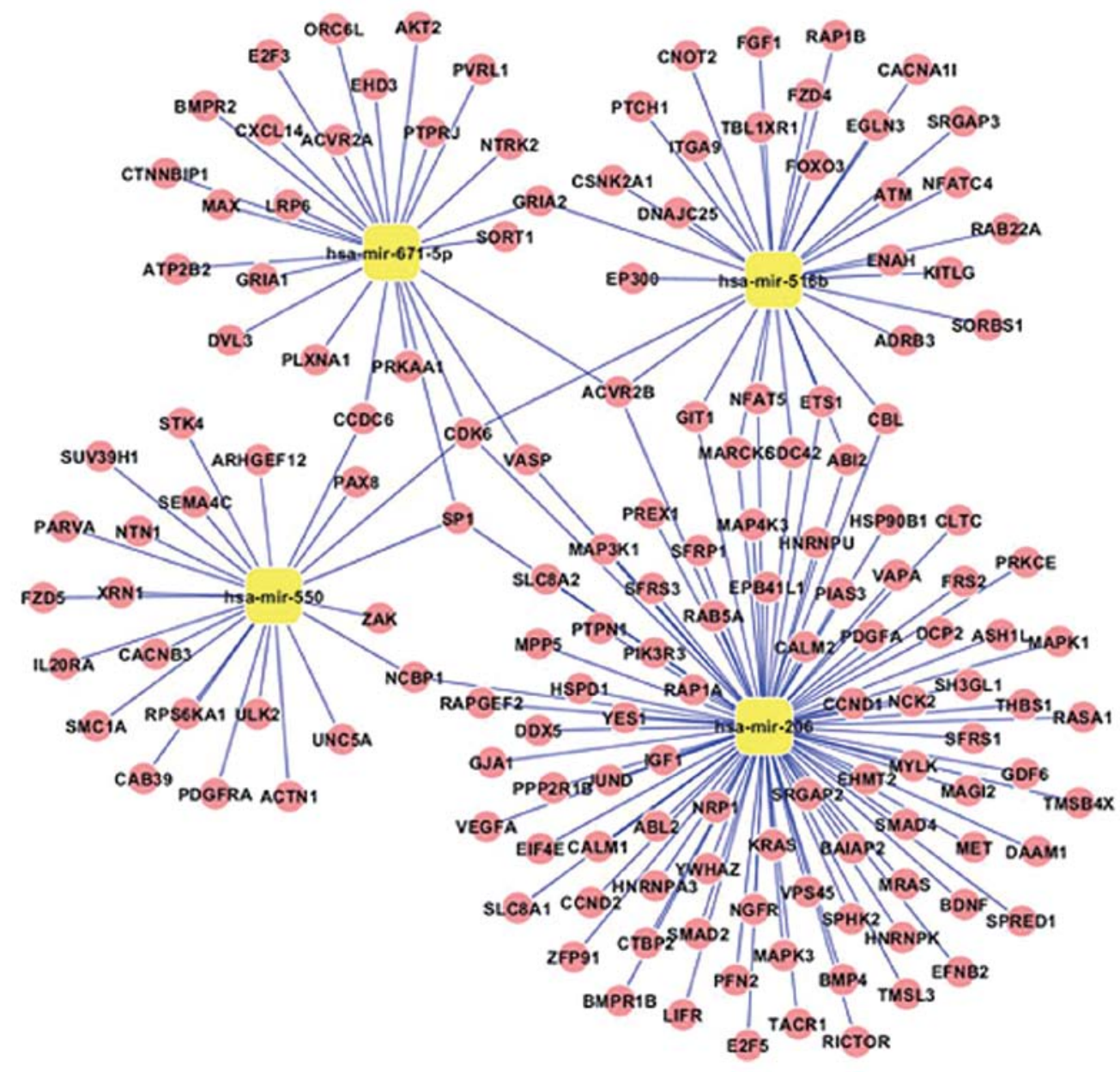

Figure 4. microRNA-gene-network. Yellow box nodes represent microRNA, and red cycle nodes represent mRNA. Edges describe the inhibitory effects of microRNA on mRNA. 
A

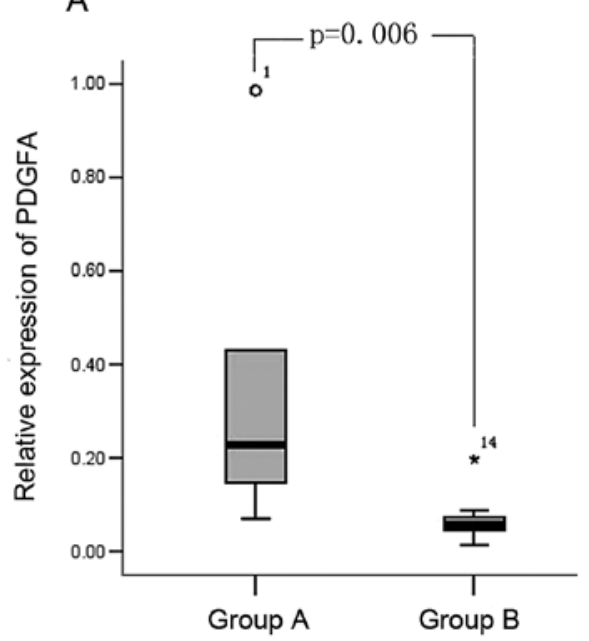

B

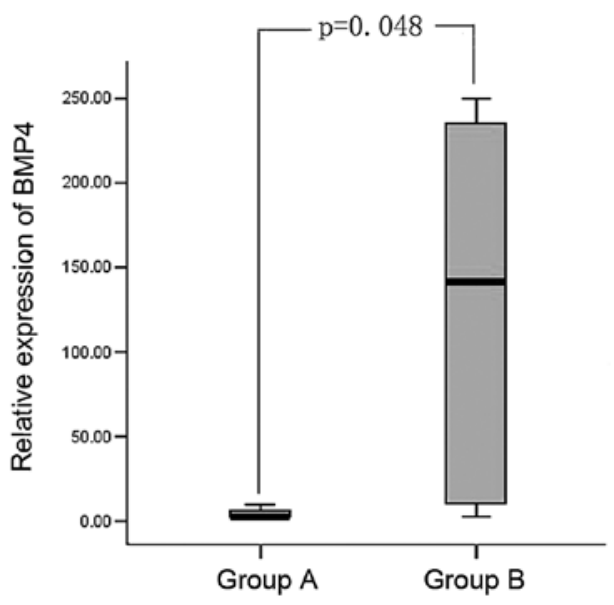

Figure 5. Two genes differentially expressed between treated and untreated prolactinomas. (A) Boxplot showing significantly different expression of PDGFA ( $\mathrm{P}=0.006)$. (B) Boxplot showing significant different expression of BMP4 ( $\mathrm{P}=0.048)$.

Table IV. Genes differentially expressed in treated and untreated prolactinomas with bromocriptine by RT-PCR.

\begin{tabular}{lccr}
\hline Genes & Group A (treated) median $(\mathrm{p} 25, \mathrm{p} 75)$ & Group B (untreated) median (p25, p75) & P-value \\
\hline PDGFA & $0.36(0.20,0.86)$ & $0.05(0.04,0.07)$ & $0.006^{\mathrm{a}}$ \\
PDGFRA & $1.61(1.15,3.33)$ & $1.68(0.08,4.05)$ & 0.839 \\
BMP4 & $1.04(1.00,8.68)$ & $136.56(7.31,231.05)$ & 0.048 \\
BMPR2 & $1.38(0.54,4.47)$ & $0.49(0.35,2.75)$ & 0.536 \\
\hline
\end{tabular}

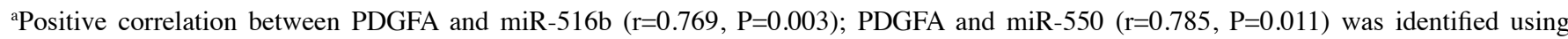
Spearman's correlation coefficient analysis.

These genes were classified into different functional categories according to Gene Ontology (GO) project for biological processes, molecular function and cellular component. The main GO categories for these genes were protein binding, binding, intracellular, positive regulation of cellular process, regulation of primary metabolic process and cell part (Fig. 3A). The KEGG pathway analysis for these putative target genes showed that the genes were involved in adherens junction, axon guidance, mTOR signaling pathway, pathways in cancer, neurotrophin signaling pathway, TGF- $\beta$ signaling pathway, MAPK signaling pathway (Fig. 3B). Genes involved in these enriched KEGG pathways and in the related GO category were selected. Additionally, miRNA-mRNA network analysis integrated these miRNAs and their putative target genes by outlining the interactions of miRNA and these selected genes (Fig. 4).

Expression of related genes of microRNAs. The role of differentially expressed microRNAs in the pharmacotherapeutic mechanism of prolactinoma. Four genes that played an important role in the TGF- $\beta$ and the MAPK signaling pathway, platelet-derived growth factor $\alpha$ polypeptide (PDGFA), bone morphogenetic protein 4 (BMP4), platelet-derived growth factor receptor $\alpha$ polypeptide (PDGFRA) and bone morphogenetic protein receptor type II (BMPR2), were selected to assess differential expression in the treated prolactinoma vs. untreated prolactinoma using the qRT-PCR assay according to the miRNA-mRNA network (Fig. 4). As shown in Fig. 5, PDGFA was significantly upregulated in treated prolactinomas compared with untreated prolactinomas $(\mathrm{P}=0.006)$ (Fig. 5A); while BMP4 was significantly downregulated in treated prolactinomas ( $\mathrm{P}=0.048$ ) (Fig. 5B). However, expression of 2 other genes, PDGFRA and BMPR2, did not show significant difference between the 2 groups (Table IV). A positive correlation was further found between PDGFA and 2 microRNAs, miR-516b and miR-550 ( $r=0.769, \mathrm{P}=0.003 ; \mathrm{r}=0.785, \mathrm{P}=0.011$, respectively).

\section{Discussion}

To the best of our knowledge, this is the first study to identify the microRNAs associated with prolactinomas treated with bromocriptine. In the present study, we confirmed that miR-206, miR-671-5p, miR-516b and miR-550 were significantly differentially expressed in treated prolactinomas versus untreated prolactinomas by qRT-PCR. Similarly, Bottoni et al found 6 microRNAs differentially expressed in treated NFPA vs. non-treated non-functioning pituitary adenoma (NFPA): 3 microRNAs (miR-134, miR-155 and miR-148) were upregulated and 3 (miR-29b, miR-29c and miR-200a) were downregulated (15). However, we did not observe the differential expression of mir-200a between pharmacologically 
treated and non-treated prolactinomas and the others were not detected by microRNA microarray.

In our study, we found that miR-206 was upregulated in treated prolactinomas. The antitumoral action of bromocriptine is connected with the induction of apoptosis (28). miR-206 identified as a pro-apoptotic activator, blocks the antiapoptotic activity of Notch3 (29). miR-206 has also been demonstrated to act as a potent tumor suppressor in c-Met-overexpressing tumors (30). Furthermore, miR-206 expression associated with genes linked to MAP kinase pathway activation (31). With regard to miR-516, previous studies have indicated that miR-206 may not only act as a tumor suppressor microRNA, but also that it downregulates metastasis-related target genes by associated DNA methylation (32) and is connected with the induction of apoptosis (28). In our study, miR-516b and miR-550 were significantly upregulated in treated prolactinomas, suggesting that these microRNAs may have a role in promoting prolactin-induced cell apoptosis. As to miR-671, it may regulate gene expression to promote tumor growth (33). In our series, miR-671-5p was significantly downregulated in treated prolactinomas in agreement with a previous report (33).

In order to gain insight into the function of these miRNAs, GO term and KEGG pathway annotation were applied to their target genes. GO enrichment and KEGG pathway analysis organized genes targeted by these 4 miRNAs into hierarchical categories based on biological processes, and then outlined the effects of miRNAs on pharmacotherapy mechanism of prolactinomas. As a result, KEGG annotation showed that important proliferative (MAPK, Wnt, VEGF and cell cycle), survival (mTOR and TGF- $\beta$ ), adhesive (adherens junction, tight junction, focal adhension and gap junction), signaling pathways were abundant among the significantly enriched pathways. The MAPK and TGF- $\beta$ signaling pathway have been reported to be involved in the subsequent pharmacotherapeutic effects of prolactinoma through inhibiting lactotroph cell proliferation and inducing apoptosis through D2 receptors (3-5). Initially, prolactinomas were treated by bromocriptine activating the $D 2$ receptor which is a $G$ protein-coupled receptor protein. Consistently, GO annotation showed that protein binding had the highest enrichment score.

The miRNA-mRNA interaction network analysis further integrated the bioinformatic findings, and displayed some critical genes of the related signaling pathway. PDGFA/ PDGFRA involved in the MAPK signaling pathway and BMP4/BMPR2 involved in TGF- $\beta$ signaling pathway were selected for further examination at the mRNA level. Data from our study shows that expression of PDGFA was significantly upregulated in treated prolactinomas. PDGFA can act directly on pituitary cells to selectively decrease the production of PRL (34). Meanwhile, PDGFA is shown to increase downstream of p44/42 MAPK phosphorylation in a dose-dependent manner (35), which results in the inhibition of cell cycle inhibitors (36). In addition, a positive correlation was found between PDGFA and 2 microRNAs, miR-516b and miR-550. An intermediate or secondary microRNA-driven regulatory mechanism, such as a transcription factor (37) may exist between miR-516b, miR-550 and PDGFA instead of a direct interaction.

Expression of BMP4 was significantly downregulated in treated prolactinomas in this study. BMP4 plays a key role not only in the initial development of the anterior pituitary (38) but also in the pathogenesis of differentiated pituitary adenoma (39). A prior study has demonstrated that bromocriptine suppresses BMP4 signaling in GH3 cells (40), and downregulation of BMP4 may suppress prolactin cell proliferation (39). Moreover, BMP4 is considered a predicted putative target gene of miR-206 by computational search algorithms. Therefore, the fact that overexpression of miR-206 in the treated prolactinomas was accompanied by downregulation of BMP4 indicates the possibility that miR-206 may inhibit tumor proliferation and induce apoptosis through the TGF- $\beta$ signaling pathway in bromocriptinetreated prolactinomas.

In conclusion, we identified 4 miRNAs, which may be involved in the pharmacotherapy mechanism of prolactinoma. Their actions may regulate multiple functions, especially protein binding. These 4 miRNAs may be involved in several signaling pathways. PDGFA and BMP4 may act as critical regulators in the MAPK and TGF- $\beta$ signaling pathway respectively. However, the detailed function associated with these miRNAs in prolactinoma pharmacotherapy needs further investigation and the target genes of miRNAs need further experimental validation.

\section{Acknowledgements}

This project was based upon work funded by the Zhejiang Provincial Natural Science Foundation of China under grant no. R2091137 and by the Zhejiang Provincial Program for the Cultivation of High-level Innovative Health talents.

\section{References}

1. Wu ZB, Yu CJ, Su ZP, Zhuge QC, Wu JS and Zheng WM: Bromocriptine treatment of invasive giant prolactinomas involving the cavernous sinus: results of a long-term follow up. J Neurosurg 104: 54-61, 2006.

2. Stefaneanu L, Kovacs K, Scheithauer BW, et al: Effect of dopamine agonists on lactotroph adenomas of the human pituitary. Endocr Pathol 11: 341-352, 2000.

3. Iaccarino C, Samad TA, Mathis C, Kercret H, Picetti R and Borrelli E: Control of lactotrop proliferation by dopamine: essential role of signaling through D2 receptors and ERKs. Proc Natl Acad Sci USA 99: 14530-14535, 2002.

4. Paez-Pereda M, Giacomini D, Echenique C, Stalla GK, Holsboer F and Arzt E: Signaling processes in tumoral neuroendocrine pituitary cells as potential targets for therapeutic drugs. Curr Drug Targets Immune Endocr Metabol Disord 5: 259-267, 2005.

5. An JJ, Cho SR, Jeong DW, Park KW, Ahn YS and Baik JH: Antiproliferative effects and cell death mediated by two isoforms of dopamine D2 receptors in pituitary tumor cells. Mol Cell Endocrinol 206: 49-62, 2003.

6. Bartel DP: MicroRNAs: genomics, biogenesis, mechanism, and function. Cell 116: 281-297, 2004.

7. Kanellopoulou C, Muljo SA, Kung AL, et al: Dicer-deficient mouse embryonic stem cells are defective in differentiation and centromeric silencing. Genes Dev 19: 489-501, 2005.

8. Croce CM and Calin GA: miRNAs, cancer and stem cell division. Cell 122: 6-7, 2005.

9. Esquela-Kerscher A and Slack FJ: Oncomirs - microRNAs with a role in cancer. Nat Rev Cancer 6: 259-269, 2006.

10. Bak M, Silahtaroglu A, Moller M, et al: MicroRNA expression in the adult mouse central nervous system. RNA 14: 432-444, 2008.

11. Farh KK, Grimson A, Jan C, et al: The widespread impact of mammalian microRNAs on mRNA repression and evolution. Science 310: 1817-1821, 2005.

12. Landgraf $P$, Rusu M, Sheridan R, et al: A mammalian microRNA expression atlas based on small RNA library sequencing. Cell 129: 1401-1414, 2007. 
13. Zhang Z, Florez S, Gutierrez-Hartmann A, Martin JF and Amendt BA: MicroRNAs regulate pituitary development, and microRNA 26b specifically targets lymphoid enhancer factor 1 (Lef-1), which modulates pituitary transcription factor 1 (Pit-1) expression. J Biol Chem 285: 34718-34728, 2010.

14. Bottoni A, Piccin D, Tagliati F, Luchin A, Zatelli MC and degli Uberti EC: miR-15a and miR-16-1 down-regulation in pituitary adenomas. J Cell Physiol 204: 280-285, 2005.

15. Bottoni A, Zatelli MC, Ferracin M, et al: Identification of differentially expressed microRNAs by microarray: a possible role for microRNA genes in pituitary adenomas. J Cell Physiol 210: 370-377, 2007.

16. Zatelli MC and degli Uberti EC: microRNAs and possible role in pituitary adenoma. Semin Reprod Med 26: 453-460, 2008.

17. Amaral FC, Torres N, Saggioro F, et al: microRNAs differentially expressed in ACTH-secreting pituitary tumors. J Clin Endocrinol Metab 94: 320-323, 2009.

18. Qian ZR, Asa SL, Siomi H, et al: Overexpression of HMGA2 relates to reduction of the let-7 and its relationship to clinicopathological features in pituitary adenomas. Mod Pathol 22: 431-441, 2009.

19. Butz H, Liko I, Czirjak S, et al: microRNA profile indicates downregulation of the TGFbeta pathway in sporadic non-functioning pituitary adenomas. Pituitary 14: 112-124, 2011.

20. Butz H, Liko I, Czirjak S, et al: Down-regulation of Wee1 kinase by a specific subset of microRNA in human sporadic pituitary adenomas. J Clin Endocrinol Metab 95: E181-E191, 2010.

21. Stilling G, Sun Z, Zhang S, et al: microRNA expression in ACTH-producing pituitary tumors: up-regulation of microRNA122 and -493 in pituitary carcinomas. Endocrine 38: 67-75, 2010.

22. Wu ZB, Zheng WM, Su ZP, et al: Expression of D2RmRNA isoforms and ERmRNA isoforms in prolactinomas: correlation with the response to bromocriptine and with tumor biological behavior. J Neurooncol 99: 25-32, 2010.

23. Xue X, Sun J, Zhang Q, Wang Z, Huang Y and Pan W: Identification and characterization of novel microRNAs from Schistosoma japonicum. PLoS One 3: e4034, 2008.

24. Sterrenburg E, Turk R, Boer JM, van Ommen GB and den Dunnen JT: A common reference for cDNA microarray hybridizations. Nucleic Acids Res 30: e116, 2002.

25. Chen C, Ridzon DA, Broomer AJ, et al: Real-time quantification of microRNAs by stem-loop RT-PCR. Nucleic Acids Res 33: e179, 2005 .

26. Huang da W, Sherman BT and Lempicki RA: Systematic and integrative analysis of large gene lists using DAVID bioinformatics resources. Nat Protoc 4: 44-57, 2009.

27. Livak KJ and Schmittgen TD: Analysis of relative gene expression data using real-time quantitative PCR and the 2(-Delta Delta C(T)) Method. Methods 25: 402-408, 2001.
28. Gruszka A, Kunert-Radek J and Pawlikowski M: The effect of octreotide and bromocriptine on expression of a pro-apoptotic Bax protein in rat prolactinoma: the effect of octreotide and bromocriptine on expression of a pro-apoptotic Bax protein in rat prolactinoma. Folia Histochem Cytobiol 42: 35-39, 2004.

29. Song G, Zhang Y and Wang L: microRNA-206 targets notch3, activates apoptosis, and inhibits tumor cell migration and focus formation. J Biol Chem 284: 31921-31927, 2009

30. Yan D, Dong Xda E, Chen X, et al: microRNA-1/206 targets c-Met and inhibits rhabdomyosarcoma development. J Biol Chem 284: 29596-29604, 2009.

31. Missiaglia E, Shepherd CJ, Patel S, et al: microRNA-206 expression levels correlate with clinical behaviour of rhabdomyosarcomas. Br J Cancer 102: 1769-1777, 2010.

32. Lujambio A, Calin GA, Villanueva A, et al: A microRNA DNA methylation signature for human cancer metastasis. Proc Natl Acad Sci USA 105: 13556-13561, 2008.

33. Lerebours F, Tozlu-Kara S, Vacher S, Lidereau R and Bieche I: microRNA expression profiling of inflammatory breast cancer. Cancer Res 69: 2009.

34. Sullivan NJ and Tashjian AH Jr: Platelet-derived growth factor selectively decreases prolactin production in pituitary cells in culture. Endocrinology 113: 639-645, 1983.

35. MacDonald TJ, Brown KM, LaFleur B, et al: Expression profiling of medulloblastoma: PDGFRA and the RAS/MAPK pathway as therapeutic targets for metastatic disease. Nat Genet 29: 143-152, 2001.

36. Grossman AB: The molecular biology of pituitary tumors: a personal perspective. Pituitary 12: 265-270, 2009.

37. Iwama $\mathrm{H}$, Murao $\mathrm{K}$, Imachi $\mathrm{H}$ and Ishida T: microRNA networks alter to conform to transcription factor networks adding redundancy and reducing the repertoire of target genes for coordinated regulation. Mol Biol Evol 28: 639-646, 2011.

38. Scully KM and Rosenfeld MG: Pituitary development: regulatory codes in mammalian organogenesis. Science 295: 2231-2235, 2002.

39. Paez-Pereda M, Giacomini D, Refojo D, et al: Involvement of bone morphogenetic protein 4 (BMP-4) in pituitary prolactinoma pathogenesis through a Smad/estrogen receptor crosstalk. Proc Natl Acad Sci USA 100: 1034-1039, 2003.

40. Miyoshi T, Otsuka F, Otani $\mathrm{H}$, et al: Involvement of bone morphogenetic protein- 4 in $\mathrm{GH}$ regulation by octreotide and bromocriptine in rat pituitary GH3 cells. J Endocrinol 197: 159-169, 2008. 